\title{
An Intelligent Framework Prototype for Monitoring Students in Virtual Classroom
}

\author{
Ashutosh Satapathy, Jenila Livingston L. M
}

School of Computing Science and Engineering, VIT-Chennai, India

\begin{tabular}{l} 
Article Info \\
\hline Article history: \\
Received Jan 12, 2018 \\
Revised Apr 21, 2018 \\
Accepted Aug 21, 2018 \\
\hline
\end{tabular}

\section{Keywords:}

Liveness face detection

Monitoring system

Virtual classroom

VoIP

VoIP cloud

\begin{abstract}
Virtual classroom is one of the fastest growing educational technologies used by many industries and institutions. Today, Voice over Internet Protocol (VoIP) cloud is become a most accepted replacement to different virtual classroom technologies such as internet chat, internet radio, web conferencing, traditional video conferencing and peer to peer VoIP calls. It provides greater flexibility, reliability and cost efficient telecommunication features and operates on comparatively lesser bandwidth. Involvement of new technology in virtual classroom makes it more reliable to teachers and students. Still it follows traditional methods to monitor students by invigilator whether it may be inside the classroom or home study. A secure framework for virtual classroom is developed to not only monitor student's activities continuously but also the amount of time spent by each student and provide security against unsecure unauthorized login. An effective monitoring System based on Liveness Face Detection techniques is proposed to add more flexibility to conduct virtual classroom and also increases the level of securities of each student's accounts.
\end{abstract}

Copyright $(0) 2018$ Institute of Advanced Engineering and Science. All rights reserved.

\section{Corresponding Author:}

Jenila Livingston L. M,

School of Computing Science and Engineering,

VIT-Chennai, India.

Email: jenila.lm@vit.ac.in

\section{INTRODUCTION}

E-learning is the use of electronic educational technologies in learning and teaching. Web based learning, multimedia learning, virtual learning environment and mobile learning are different modes of providing education [1]. It is mainly divided into two types, asynchronous e-Learning and synchronous e-Learning. Synchronous e-Learning is the online class that requires both students and teacher to be online at the same time. Virtual classroom (VC) is the virtual learning environment, where teacher and students are logged into it at the same time and participate in synchronous instruction just like real-world classroom. It can be web based (e.g., BigBluebutton) or software based (e.g., Skype). In web-based VC, web browsers are used as classroom interface and it is platform independent [2]. In software based VC, light weight applications are used as classroom interface which is platform dependant i.e. applications format and size are varied for different end user operating systems.

As discussed earlier, virtual classroom enables teacher and students to come online to take part in classroom session. It not only connects students from different corner of the earth, but also allows them to see and talk with each other during classroom session. Different technologies are involved to setup virtual classroom [3]. Some of the new technologies are discussed below. 


\subsection{Virtual Classroom Technologies \\ 1.1.1 Virtual World}

Virtual world education provides 3-D environment where teacher and students meet alive and interact with each other. It uses new generation communication technology VoIP and digital equipments for better communication and greater display. As systems are equipped with large memory, storage, high power computational units (e.g., graphics card, CPU), the bandwidth consumption is extremely high.

\subsubsection{VoIP Cloud}

VoIP cloud communication is IP network based audio and/or video communications where communication applications, processors, memories and storages are hosted at a third party datacentre and these are accessed over the internet [4]. Particularly, VoIP cloud communication is the successor to typical point to point VoIP. Here, teachers and students login using thin terminals to access cloud services.

\section{LITERATURE REVIEW}

Most of the employees are willing to pursue a second degree after earning a bachelor degree. Most of degree students are aspiring to boost their careers by taking courses in the form of distance education. The most important issue in distance education is student's attitudes and teacher's preparedness. It requires distance-learning courses are needed to be properly implemented and delivered. So that student can learn as much as in on-campus courses [5]. The USDLA stated that telecommunication technologies can be used to deliver teaching in distance learning environment, which transmit and receive various stuff through voice, video and data. To address the above issue, virtual classroom is widely adopted around the world in last two decades. One of the major issues related to virtual classroom is the roles and activities of participants. It includes a great deal of resources dedicated to teaching preparation and student's support [6].

Harris described, the challenges a teacher faces during virtual classroom related to monitoring student's activities. Students are often distracted by the availability of the technologies such as email, surf the internet etc. As internet open the door to the information outside the classroom, there is an increase the possibility of academic dishonesty. A tool named SynchronEye which was used by the teacher to observe student's activities by providing real time view of student's monitor as thumbnails or a full screen [7]. It allows teacher to broadcast selected screens to remote students to prompt collaborative learning.

Jakab et al. presented a unified communication technology that integrates several communications and supports services such as voice, email, web form, white board and file sharing [8]. Most used protocols and architectures for unified communication technology are directly from VoIP solutions. It provides contact on the basis of the communication canal with currently highest priority. If somebody wishes to communicate with such person, its accessibility could be checked through communication canal. Video conference is done through conference server by changing end point equipment to video VoIP phone. This framework is not resistive against unsecure unauthorized login using credentials of registered student.

Donda et al. proposed a distance education system has two components, a classroom system and remote student system [9]. Classroom system is extended in such a way that remote students are transparent to teachers and classroom students. The classroom system and the remote student system use a common framework for media communication. The communication framework uses a group communication system (GCS) that enables reliable and efficient communication between teachers and students logically. GCS provides services such as FIFO ordered message delivery, group membership etc. It is unable to provide attendance for registered students. Here, instructor is unable to monitor behavior of both local and remote students at the same time.

Dehnavi explained a new web based e-learning model with attendance control ability, which uses multimodal biometric characteristic (facial features) and behavioral biometric characteristics (keystrokes and mouse movements) to track student's activities [10]. A new algorithm, attendance control tracker was presented to demonstrate use of these biometrics properties. Experimental results show that, above attendance control system also worked well with student collaboration work.

Potnis and Jahagirdar proposed a HAAR cascade classifier was used for real time face detection system for virtual classroom to reduce time taken for classification and achieve desired outcome [11]. The results show the average CPU and memory uses were significantly low compare to other similarly existing system.

Since 2009, Hanoi Open University (HOU) has been providing live virtual classes for various learning programs. Trung et al. discussed different types of multimedia-enabled delivery approached, their issues and the technologies behind them [3]. Overall, VoIP cloud has found to be efficient in synchronous eLearning such as virtual classroom, project collaboration and share inspiration compare to web conferencing, traditional video conferencing, internet radio and virtual world. It also consumes significantly less bandwidth and provides least overhead to end user. 
Saha presented the different types of video conferencing and virtual classroom services developed over NICNET/ NKN by National Informatics Center (NIC) [12]. It explains two types of video conferencing systems named dedicated systems and cloud based desktop, are used widely in public and private sectors in India. It contains silent features of cloud based desktop video conferencing which makes it popular over dedicated system based video conferencing. At last, it explains two types of virtual classrooms named typical high end virtual classroom and web based virtual classroom are widely used in most of the public/private schools and universities. The web based virtual classroom is gaining popularity over typical high end virtual classroom because of less consumption of bandwidth and its cost effectiveness.

In virtual classroom, students and teacher are logged into learning environment to start online class. Still universities follow traditional method to monitor university students by invigilator during virtual classroom learning which waste of time and resources. Still, monitoring home students is really a big challenge. Bring machine instead of human being may solve this problem in future. Here, it is used to monitor liveness facial features (diffusion of light, facial movement) to make a decision continuously. The complete framework of monitoring system is discussed below.

\section{VOIP CLASSROOM FRAMEWORK}

\subsection{VoIP}

VoIP communication supports audio and/or video communication over IPv4 and IPv6 networks [13]. It is completely divergent from the circuit-switched telecom network. In circuit switched telephone network, resources are assigned to individual call separately and the connection path is static for each call from beginning to end. T carriers, Public Switch Telephone Network (PSTN), local loops, Plain Old Telephone Service (POTS) and dial up are some of the traditional services under International Telecommunication Union (ITU) and they are used for voice telecommunication. VoIP supports various signaling protocols; it may be open standard or proprietary protocol. VoIP communication system is mainly divided into user end (call manager, mail, soft phone and dashboard etc.), machine end (CPU, GPU, memory, storage, network and operating system etc.) and middleware (VoIP protocols, DHCP server, DNS server, web server and database server etc.) [14]. Its communication layer consists of five layers as shown in Figure 1.

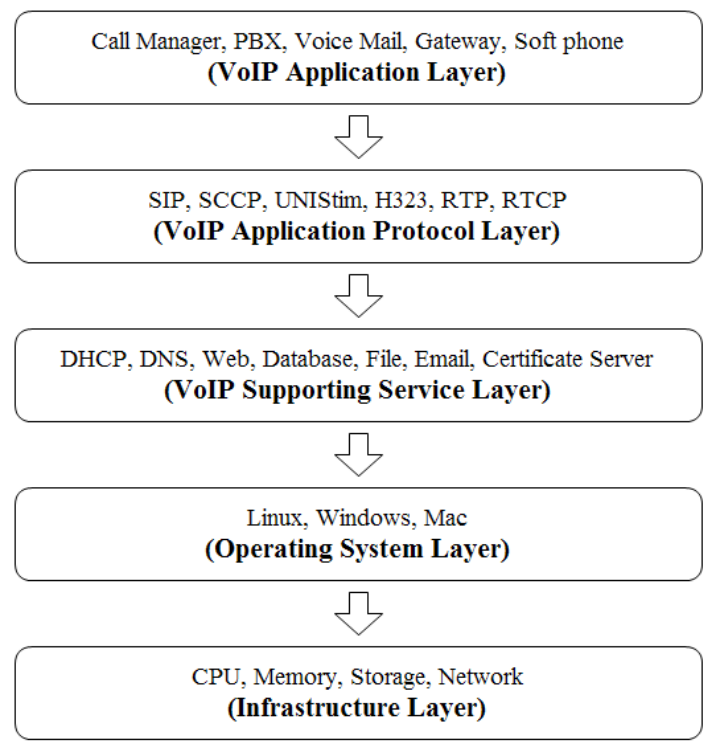

Figure 1. VoIP layers

\subsection{VoIP Cloud}

VoIP cloud communication is the technology behind telephony services which provide access to resources of data center through a light weight terminal. The terminal may be web based or software based applications [15]. Users store their information which is distributed across multiple servers inside the cloud. Apart from the above, VoIP cloud has features such as reliability, elasticity, scalability, on demand services, 
pay as you go, resource pooling and resource optimization [16-17]. The twelve main components of VoIP cloud are shown in Figure 2. Some of the main components are discussed below.

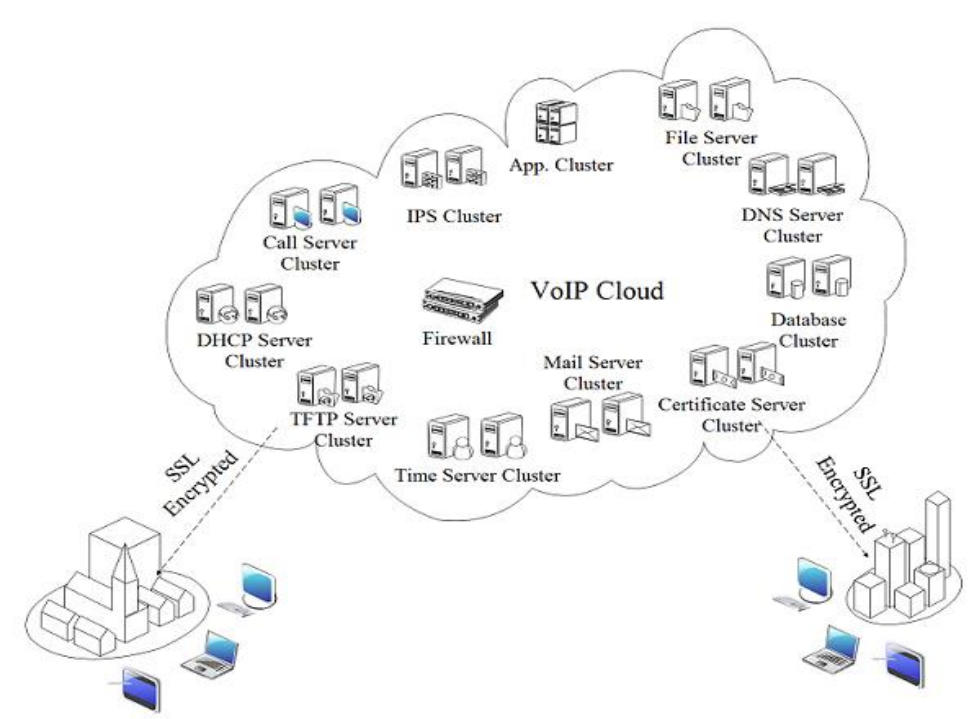

Figure 2. VoIP cloud architecture

\subsubsection{Call Server}

During phone setup, all the endpoints are registered with call server. It populates interfaces with information about topology configuration and signaling protocol which provide feature support to endpoints. It also provides security and controls the connection during call services. All the real time data packets carried by transport protocol are passed through call server.

\subsubsection{DHCP Server}

It provides IP addresses to cloud internal network elements and reserves IP addresses for different servers. It also provides IP address of default gateway, primary and secondary DNS servers. A DHCP server is valid within its network i.e. DHCP server of VoIP cloud internal network is different from DHCP server of student's or teacher's network.

\subsubsection{TFTP Server}

TFTP file contains information such as IP address of call server, defined tune and dial pattern for a particular network. It not only helps to update switches, routers, firewalls and phones firmware but also provides setup files those contain configuration parameters for VoIP network e.g., codec, phone provisioning, VLAN and QoS information.

\subsubsection{Intrusion Prevention System}

Intrusion Prevention System (IPS) monitors system or network activities for any malicious or policy violation instances and takes decision based on predefined rules. It is of two types, host based IPS and network based IPS. Network based IPS monitors activities occurring inside a network where as host based IPS monitors activities occurring on a single host.

\subsection{VoIP Cloud Classroom}

VoIP cloud virtual classroom is one of the synchronous learning environments where teachers and students interact with each other at the same time. It allows both university students and home students to take part in classroom learning. Here, students learn directly from teacher and ask questions if there are any doubts. Each student sits in front of a PC attached with webcam that captures video and sends it to VoIP cloud. The Call servers sitting inside cloud forwards this to teacher's PC. This video is displayed in a frame inside the dashboard. Like this, multiple students are displayed in separate frames inside the teacher's dashboard. Student's dashboard displays teacher and his presentation in two different frames. Apart from displaying video, dashboard also provides features such as texting, Email, collaboration and document sharing [18]. VoIP virtual classroom structure as shown in Figure 3. 

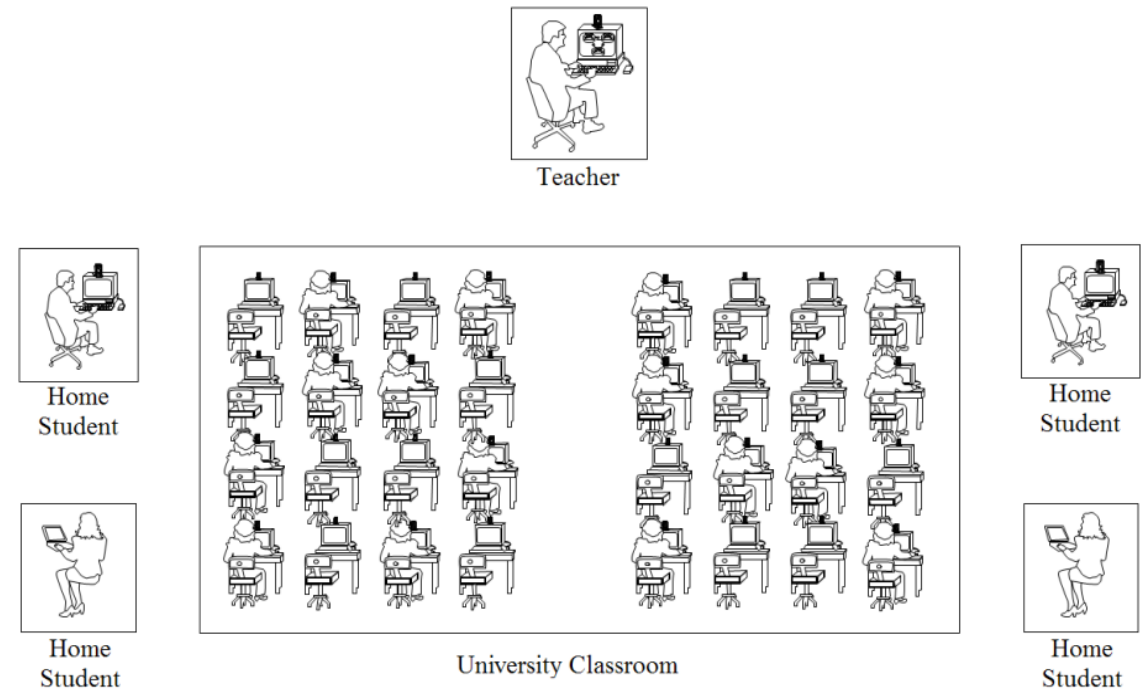

Figure 3. VoIP virtual classroom structure

\section{PROPOSED MODEL}

The monitoring system is one kind of IPS, used to monitor application data (lives facial features). Effective IPS architecture using Liveness Face Detection (LFD) technique is proposed for virtual classroom in VoIP cloud environment. Here, teachers and students are login to VoIP cloud to start class as shown in Figure 4. Both audio and video are passed through VoIP cloud network.

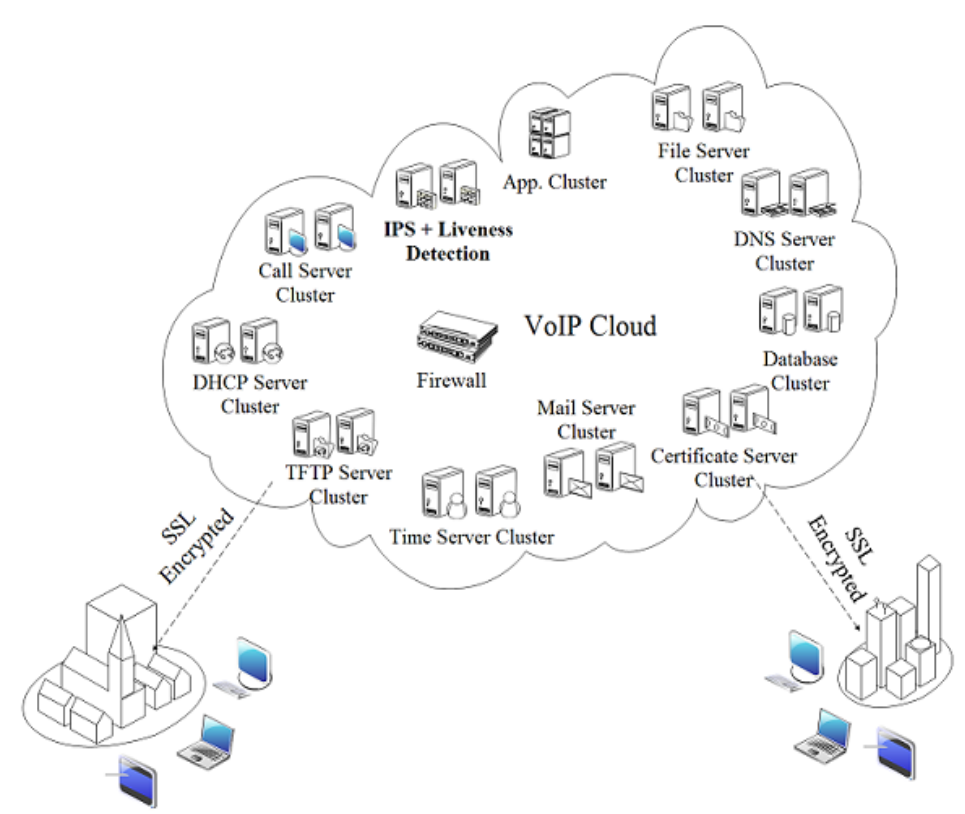

Figure 4. Proposed VoIP cloud architecture for virtual classroom

IPS are also called as intrusion detection and prevention systems. The main tasks of intrusion detection and prevention system are to identify abnormal activities, log all necessary information about the activities and try to block/stop them. A host based detection/prevention system is only concerned with monitoring the system activities, those are happening on a single host and attempt to take necessary actions against it. A network based intrusion prevention system is only concerned with data flowing through the network. 


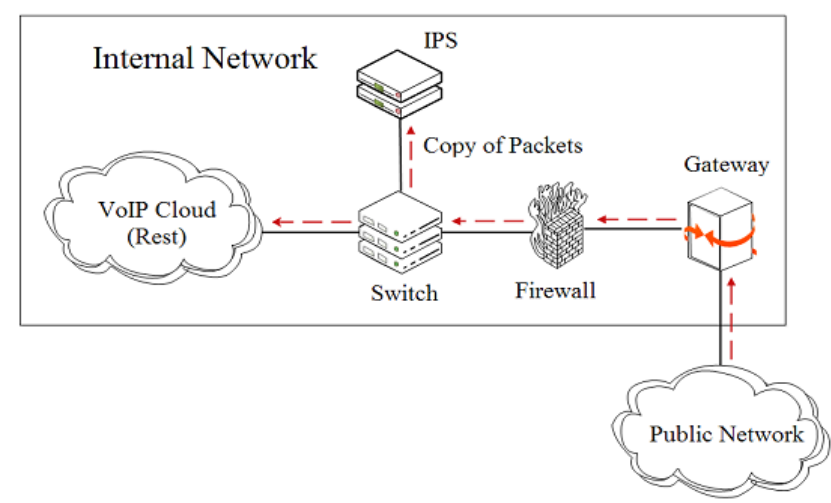

Figure 5. Proposed IPS network architecture

As VoIP communication handles real time data (audio and video), deployment of IPS plays an important role to make the service effective and efficient. Video data packets are flowing from the public network to the cloud private network will be duplicated for IPS. In pass-by configuration, a copy of the traffic is sent to the IPS while the original data travel to and from one network from and to another network as shown in Figure 5. Corresponding image frames are constructed from duplicate packets at IPS. It is a continuous process. If the IPS identifies any disturbances in video frames, it will log/record the activities and send warning message to students. Exceeding the threshold, it will close the connection of the corresponding students.

During virtual classroom, each student must be allotted to separate system embedded with webcam which captures video (sequence of frames) and forward them to cloud. The monitoring system sitting inside cloud duplicates the data and started monitoring using LFD technique. The aim of the LFD technique is to provide security to student accounts by allowing only active genuine person to successfully login. In addition to that, whether the registered students are attending the class, if yes, how much time is spent by each student? It also monitors the activities of the students in virtual classroom. The working principle of monitoring system is divided into two stages as shown in Figure 6.
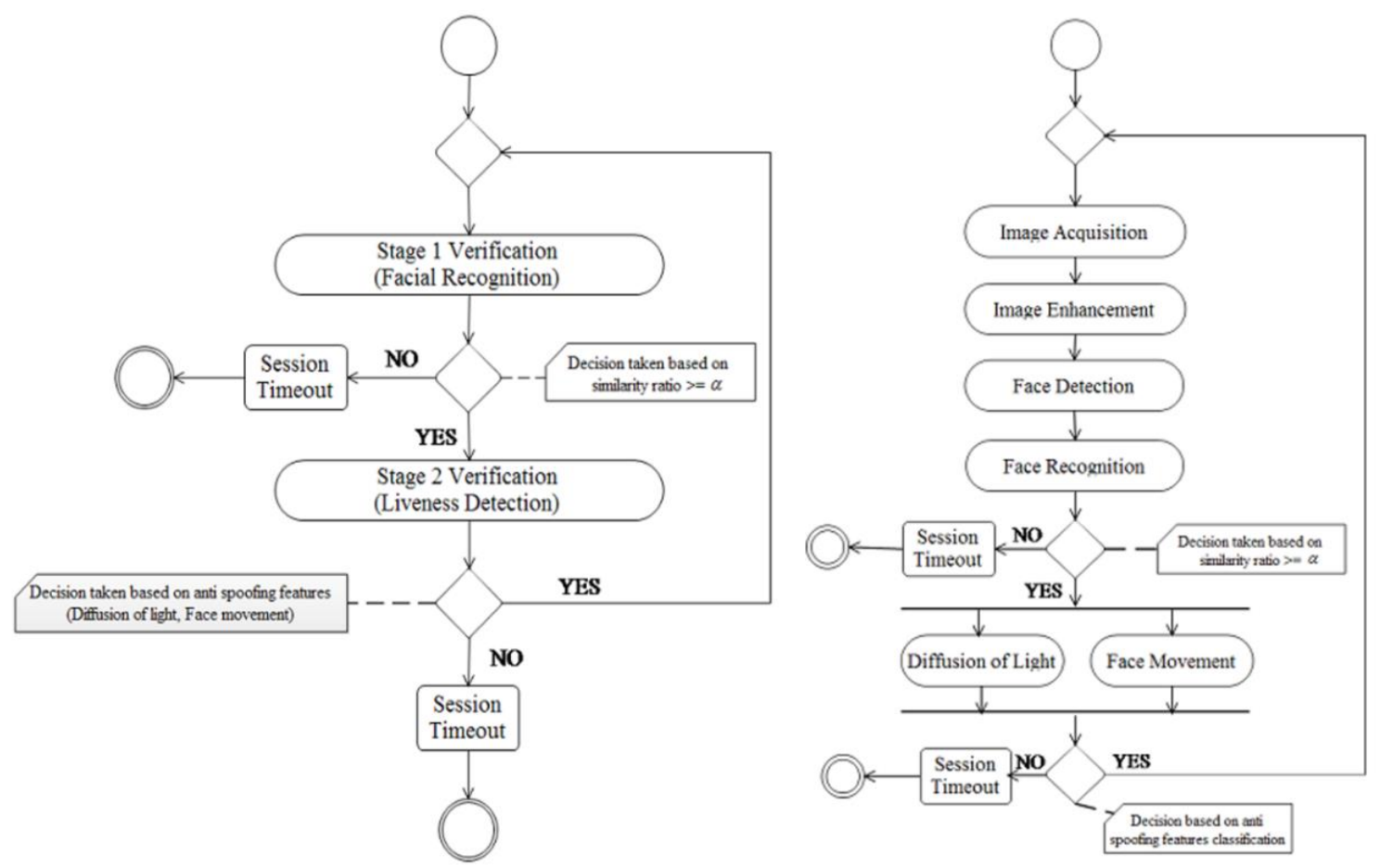

Figure 6. Work model of monitoring system (left side) and its different modules (right side) 


\subsection{Two Stages of LFD}

\subsubsection{Verification (Facial Recognition)}

Extracted features from face of the students are verified using classifier. The classifier was trained using array of features, extracted and stored in database separately at the time of registration. After passing the stage 1 verification, it moves to stage 2 verification. Otherwise connection will be blocked by firewall as per the instructions given by the monitoring system.

\subsubsection{Verification (Liveness Face Detection)}

Anti-spoofing features (diffusion of light throughout face and facial movement) are extracted from the captured face images of student and verified using classifiers. Liveness face detection technique is mainly classified into two processes which operate simultaneously as shown in Figure 6. One is liveness detection based on diffusion of light throughout face to find out student's face is fake or not i.e. only live students are allowed to attend the class. Other one is facial movement which is used to monitor the activities of students i.e. attentive or not or whether student is sleeping while class is going on. At last, both stage one and stage two verification strengthen the level of security of each student account. All the modules of work model are discussed below.

Step.1. Image Acquisition: From video captured by webcam, frames are extracted which are given as input to the next module of work model.

Step.2. Image Enhancement: Monitoring system running inside the VoIP cloud environment captures replicated packets generated by ingress switch and goes for reduction of noise and improves the interpretability or perception of information in images to provide better input for the next level. It will be achieved by smoothening and sharpening the visual data.

Step.3. Face Detection: Real time face detection on motion analysis. It normalizes distinct facial features extracted from frame and given to next step for recognition. Bounding boxes are used to identify faces in frames.

Step.4. Face Recognition: In face recognition process, student authenticity is checked. If No, the session will be timeout after a number of continuous successful recognition. If yes, it will go for the next level verification.

Step.5. Features Extraction (anti spoofing features): After face successfully recognized, it goes for extracting anti spoofing features such as diffusion of light throughout face and movement of face [19-20]. From diffusion of light, local patterns of diffusion speed are extracted which is significantly different for live and fake faces. From face movement, the deviation from normal movement of face is extracted. Both features are used in liveness detection module for final stage verification.

Step.6. Liveness Face Detection: Local patterns of diffusion speed are used to detect whether the captured face image is from genuine students or not. It will be helpful for giving attendance to registered students and how much time attended by each student. From face movement, the deviation from normal movement is calculated which is filtered students by their behavior (attentive or sleeping). These six steps are repeated for all the frames of student's video captured from respective environment.

Monitoring system using liveness face detection must be implemented in distributed environment. Hyper threading and GPU computing are introduced for parallel processing i.e. each module work is given to different thread to make these operations much faster.

\section{CONCLUSION}

From last two decade, virtual classroom is one of the widely adopted technologies in educational and industry sectors. This research paper discuss, different methods / models were adopted by researcher to add more flexibility and reliability to learning environment. The rise of VoIP cloud makes it more popular, which uses IP network as its backbone. Still these follow traditional way of monitoring students by invigilator which is waste of time and resources. In this paper, an effective VoIP cloud framework for virtual classroom is proposed which uses LFD techniques to monitor student's activities. The key element, pass-by IPS has no effect on quality of service as its woks on duplicate data. At last, more research has to be done to improvise synchronous learning environment in all aspects.

\section{REFERENCES}

[1] Tahir ZM, Haron H, Singh JKG. "Evolution of Learning Environment: A Review of Ubiquitous Learning Paradigm Characteristics". Indonesian Journal of Electrical Engineering and Computer Science (IJEECS). 2018; 11(1): 175181.

[2] Veeramanickam MRM, Radhika N. “A Smart E-Learning System for Social Networking”. International Journal of Electrical and Computer Engineering (IJEECS). 2014; 4(3): 447-455. 
[3] Trung NT, Thu TTL, Tan LM. "Replacing Face-to-Face Classes by Synchronous Online Technologies: The HOU Experience". Third International Conference e-Learning. Bandung, Indonesia. 2011: 386-392.

[4] Gerea F, Ghencea DA. "Management Strategies for Integrating Cloud Computing into VoIP”. Journal of Trends in Computing and Information Sciences. 2012; 3(8): 1195-1199.

[5] Gagne M, Shepherd M. "Distance in Accounting". THE Journal. 2001; 28(9): 58-64.

[6] Hartman J, Lewis JS, Powell KS. "Inbox Shock: A Study of Electronic Message Volume in a Distance Managerial Communication Course". Business Communication. 2002; 65(3): 9-28.

[7] Harris RB. "Monitoring Student Activities in a Computer Classroom - NIFTY TOOLS". Journal of Computing Science Colleges. 2007; 23(1): 107.

[8] Jakab F, Jakab M, Janitor J, Reves M. "Communication Challenges in e-Learning Solutions". Fifth International Conference on Emerging e-Learning Technologies and Applications. High Tatras, Slovakia. 2007: 1-5.

[9] Dondera D, Jia C, Popescu V. "Virtual Classroom Extension for Effective Distance Education". IEEE Computer Graphics and Application. 2008; 28(1): 64-74.

[10] Dehnavi MK, Fard NP. "Presenting a Multimodal Biometric Model for Tracking the Students in Virtual Classes". Procedia Social and Behavioural Science. 2011: 3456-3462.

[11] Potnis SS, Jahagirdar AS. "Real-time Face Detection System for Virtual Classroom Environment". International Journal of Research in Computer and Communication Technology. 2014; 3(7): 749-752.

[12] Saha D. "Video Conferencing and Virtual Classroom Services over NICNET/NKN". NIC. New Delhi, India. 2016.

[13] Hartpence B. "Introduction to Voice over the Internet Protocol". Packet Guide to Voice over IP. Sebastopol, CA. O'Reilly; 2013: 1-34.

[14] Satapathy A, Livingston LMJ. "A Comprehensive Survey of Security Issues and Defense Framework for VoIP Cloud”. Indian Journal of Science and Technology. 2016; 9(2): 1-13.

[15] Patinge SA, Soni PD. "Metamorphosis in VoIP Cloud Computing Services used in VoIP”. International Journal of Application Innovation in Engineering Management. 2013; 2(2): 236-239.

[16] Mahmood Z. "Cloud Computing: Characteristics and Deployment Approaches". Eleventh IEEE International Conference on Computer and Information Technology (CIT). Pafos, Cyprus. 2011: 121-126.

[17] Shyamala K, Rani TS. "An Analysis on Efficient Resource Allocation Mechanism in Cloud Computing”. Indian Journal of Science and Technology. 2015; 8(9): 814-821.

[18] Nadesh RK, Aramudhan M. "An Empirical Study on Peer-to-Peer Sharing of Resources in Mobile Cloud Environmwent". International Journal of Electrical and Computer Engineering (IJECE). 2018; 8(3): 1933-1938.

[19] Kim W, Suh S, Han JJ. "Face Liveness Detection from a Single Image via Diffusion Speed Model". IEEE Transactions on Image Processing. 2015; 24(8): 2456-2465

[20] Jung H, Lee S, Park S, Kim B, Kim J, Lee I, Ahn C. "Development of Deep Learning-based Facial Expression Recognition System". Twenty First Korea-Japan Joint Workshop on Frontiers of Computer Vision. Mokpo, South Korea. 2015. 